\title{
A future up in the air
}

\section{Helium has become a precious resource and its supply must be managed with care.}

For physicists, helium is often synonymous with low-temperature physics - both as a cryogen and a research material itself. It is possible to reach a base temperature of $4.2 \mathrm{~K}$ using liquid ${ }^{4} \mathrm{He}$; a ${ }^{3} \mathrm{He}$ cryostat can reach $0.3 \mathrm{~K}$ with pumping; put the two isotopes together in a dilution refrigerator and temperatures down to $2 \mathrm{mK}$ become routinely obtainable. Both helium species also support superfluid phases: superfluid ${ }^{3} \mathrm{He}$ is especially interesting as a quantum fluid; a supersolid phase is sought in ${ }^{4} \mathrm{He}$.

For years, there was no question of the availability of ${ }^{3} \mathrm{He}$, and prices were nearly constant at US\$100 per litre. ${ }^{3} \mathrm{He}$ is a decay product of tritium, an isotope of hydrogen, which was produced in abundance during the nuclear arms race. Although the US has since cut back on tritium production, its stockpile was capable of meeting the annual ${ }^{3} \mathrm{He}$ demand of roughly 8,000 litres. ${ }^{4} \mathrm{He}$ exists in large underground deposits around the world. (For the full story on ${ }^{4} \mathrm{He}$, see W. P. Halperin's Commentary on page 467 of this issue).
But after 11 September 2001, in the interest of national security, the US government started using ${ }^{3} \mathrm{He}$-based neutron detectors ('radiation portal monitors') to uncover any potential bombmaking components entering the country. That increased demand, coupled with a large order from the Oak Ridge National Laboratory in 2008, suddenly revealed that the US Department of Energy was allocating ${ }^{3} \mathrm{He}$ faster than it could be produced. In 2008, the demand reached 80,000 litres, becoming unsustainable (D. A. Shea and D. Morgan, Congressional Research Service, 2010; http://go.nature.com/Lx7Va5).

By September 2009, according to the report by Shea and Morgan, the US Departments of Energy, Homeland Security and Defense together decided to stop using ${ }^{3} \mathrm{He}$ in radiation portal monitors and that further allocations would be determined by three criteria: "whether alternatives to ${ }^{3} \mathrm{He}$ exist for the planned application; whether the application increases national or homeland security; and whether the required ${ }^{3} \mathrm{He}$ is needed to complete prior investments in infrastructure".

The allocation of ${ }^{3} \mathrm{He}$ has become a tricky balancing act. Although the importance of national security cannot be disputed, investment in scientific infrastructure would be wasted if equipment can't be operated. We must find alternatives, reduce or postpone demand and locate new sources.

There is plenty of ${ }^{3} \mathrm{He}$ in the Universe: the gas giants have a large supply from the original solar nebula and there are reserves on the Moon. In practical terms, heavy-water reactors from which tritium is regularly removed and stored are promising sources. Helium is also found in natural gas.

Scientists are also adapting, using cryogen-free technology that (although prone to vibration) is compatible with dilution refrigerators and superconducting magnets. This is one of many innovations that will address - and hopefully solve - the helium problem.

\section{It's good to share}

\section{Datasets can now be published, shared — and cited — in Scientific Data.}

Last month, Nature Publishing

Group welcomed its newest journal, Scientific Data - a peer-reviewed, openaccess publication designed to provide a better way to share and explain data. Scientific Data publishes Data Descriptors, a new article type that allows authors to describe important datasets in detail, making them more accessible and more easily reusable. Importantly, Data Descriptors help to give the credit deserved to the scientists who share.

Because Scientific Data articles do not contain extensive interpretative analysis or new findings, they can be used to publish a wide range of valuable datasets. All publications are released immediately under one of three Creative Commons licences, supported by an article processing charge paid by authors. The actual data files are hosted at external repositories under open terms, and Scientific Data works with authors to find the best repository for each dataset. In addition, publications at Scientific Data have unique features that are designed to maximize the discoverability and reusability of each published dataset, such as formal data citations and curated machine-readable metadata to aid advanced users and data miners.

Scientific Data has been initially developed in close collaboration with data-sharing advocates in the life and environmental science communities, and its first publications reflect these roots. However, the concepts that underlie Scientific Data - promoting reproducible, collaborative science and giving due credit to scientists - have broad currency across scientific disciplines.

The editorial team of Scientific Data is now exploring how it can best serve the physical sciences by learning about the particular datasharing challenges faced by each community.

Indeed, the physics community has its own, unique history of promoting sharing - notably the arXiv preprint server, used extensively for more than twenty years by physicists in various disciplines. Some major projects routinely share outputs, such as the NASA missions that make their data publicly available following a short period of private analysis by the mission scientists. But many other important datasets, particularly from smaller-scale projects or individual labs, are not normally shared.

Nature Physics, like all Nature journals, encourages the sharing of data and materials; our policy stipulates that, where requested, these be made "promptly available to others without undue qualifications". Scientific Data complements this policy by rewarding authors who are willing to go above and beyond our standards - for example, by providing more in-depth descriptions and fuller release of important datasets already analysed in previous publications.

We feel that Scientific Data will be just as relevant and valuable in the physics community as it is elsewhere, and invite physicists who are interested in promoting the sharing of data to get in touch with the Scientific Data team: scientificdata@nature.com. Share with us your ideas for how we might shape this concept of data publication, to best work for our community. 